\title{
PAPER
}

\section{Novel methods of enhanced endoscopic imaging}

\section{J Van Dam}

Gut 2003;52(Suppl IV):iv12-iv16

Endoscopy has become an essential part of the practice of gastroenterology. Techniques exploiting previously unused properties of light have demonstrated the potential to enhance the ability to make clinical diagnoses without removing tissue as has been standard practice for decades. The term used for many of these techniques is "optical biopsy" and, although not yet widely available, enthusiasm for such techniques has grown as has research in their potential clinical utility.

\section{FLUORESCENCE SPECTROSCOPY}

Tissue spectroscopy is based on the evaluation of characteristic patterns of light emission or reflection from tissue. Laser induced fluorescence (LIF) spectroscopy uses laser energy to stimulate endogenous tissue fluorophores to emit light (fluoresce). ${ }^{1-6}$ Determining which fluorophores within an epithelium are responsible for differences in fluorescence emission between dysplastic and non-dysplastic tissue has remained speculative. Collagen is a major fluorophore in both normal and dysplastic colonic mucosa. However, by exciting frozen sections of colonic tissue with light wavelengths of 351-364 nm, Romer and colleagues demonstrated that the cytoplasm of dysplastic tissue contained a significantly increased amount of some unidentified fluorophore, perhaps of a porphyrin derivation. ${ }^{6}$ The intensity of tissue fluorescence correlated directly with the degree of dysplasia, suggesting the increasing presence of this fluorophore as cells became more poorly differentiated. In patients with Barrett's oesophagus, LIF spectroscopy has been applied to the detection of dysplasia and carcinoma. ${ }^{45}$ One recent study reported that LIF spectroscopy was able to detect osophageal cancer (both squamous cell and adenocarcinoma) with a sensitivity of $100 \%$ and specificity of $98 \% .{ }^{4}$ While the exact causes of differences in fluorescence eludes investigators, the observed differences in fluorescence forms the basis for using fluorescence spectroscopy for distinguishing normal from dysplastic tissue.

Initial experience with spectroscopy involved the evaluation of small regions of tissue via thin, through the scope optical probes brought into light contact with the tissue. These devices were not designed for the surveillance of large surface areas (as in Barrett's oesophagus or chronic ulcerative colitis). Recently, however, spectroscopic technology has been incorporated directly into the imaging system of a flexible endoscope (that is, a "spectral endoscope") for use in the gastrointestinal tract. ${ }^{78}$ This development may well surmount most technical limitations of this technology. Future studies will determine the clinical impact of this enhanced imaging technique in larger scale prospective studies.

\section{LIGHT SCATTERING SPECTROSCOPY}

Light scattering or reflectance spectroscopy (LSS) uses an analysis of the intensity and wavelength of light reflected from the surface of a given tissue to estimate the size and degree of crowding of surface epithelial nuclei. ${ }^{9}$ The reflectance of light from a tissue results from two properties of light that are inherent to a given tissue: absorption and scattering. Absorption depends upon the tissue concentration of specific biochemicals, such as haemoglobin, which absorb particular wavelengths of light while reflecting all others. Scattering depends upon the size and density of space occupying structures such as collagen, nuclei, and other organelles in the tissue being studied. Scattering occurs as photons of light interact with and pass through these structures. Most information obtained from studying the bulk scattering component of reflectance represents information about the presence of large particles, such as the presence of collagen in the lamina propria and submucosa. ${ }^{10}$ The results of light scattering may be determined mathematically by using Mie scattering theory. This theory predicts that a spectrum from scattered light carries information about two key variables: the size distribution and refractive index of the scatterer. $^{11}$ This mathematical model requires assuming a range of particle size in the tissue a priori. The range may be broad enough, for instance 0.35 to $1.5 \mu \mathrm{m}$, to represent typical biological sizes of nuclei and cellular organelles. Measuring reflectance then permits the determination of both scatterer size and density. Coupled with the absorbance of particular wavelengths, reflectance spectroscopy can provide insight into the biochemical and structural nature of a tissue. This technique generally uses white (non-laser) light. ${ }^{10}$ Such analyses of cellular size and crowding may have important clinical applications. A second mathematical method for use in LSS is to perform a Fourier transformation. This analysis is based upon a characteristic oscillatory behaviour of scattered light based on the interference of light as it travels through cells. These oscillations are directly proportional to the size of the scatterer. If there is a range of sizes of scatterers (that is, cell nuclei), this distribution can be determined based on a mathematical method called a Fourier transformation.

Abbreviations: LIF, laser induced fluorescence; LSS, light scattering spectroscopy; OCT, optical coherence tomography 


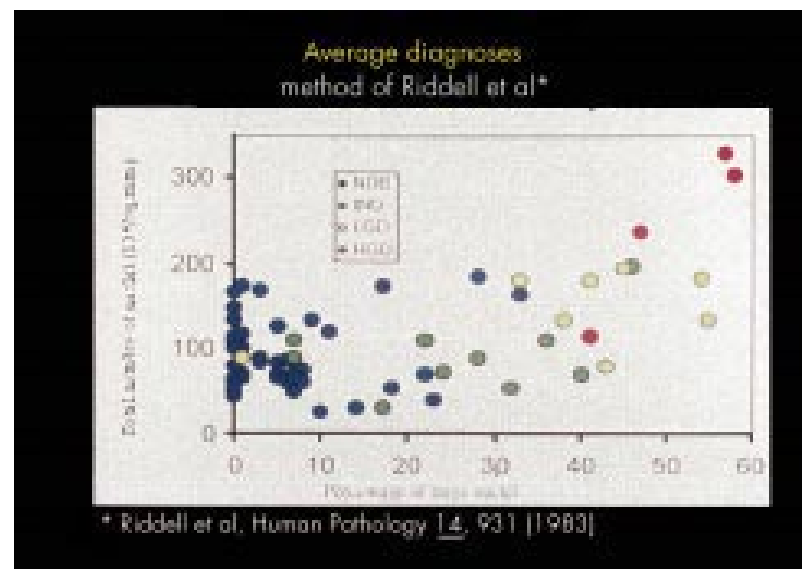

Figure 1 The degree of dysplasia (NDB, non-dysplastic Barrett's; IND, indeterminate for dysplasia; LGD, low grade dysplasia; HGD, high grade dysplasia) in 13 patients with Barrett's oesophagus. Colour diagnosis indicates the average diagnosis of four gastrointestinal pathologists blinded to results obtained by light scattering techniques. Data from reference 12: Gastroenterology 2000;119:677-82.

In patients with Barrett's oesophagus, enlargement and crowding of cell nuclei are morphological changes that signal the progression from benign metaplasia through dysplasia to cancer. In a recent study using LSS to evaluate 13 patients with Barrett's oesophagus, 76 biopsy sites were studied..$^{12}$ Four of the sites were from areas of high grade dysplasia, eight sites were of low grade dysplasisa, 12 sites were indefinite for dysplasia, and 52 sites were from non-dysplastic Barrett's mucosa. The degree of dysplasia diagnosed by histological examination was compared with the percentage of enlarged nuclei detected at each site by LSS (fig 1) The more dysplastic lesions not only had a significantly greater percentage of enlarged nuclei, but an algorithm based on LSS findings could predict the presence of dysplasia with $90 \%$ sensitivity and specificity. This preliminary in vivo clinical experience suggests that spectroscopic analysis may enhance the detection of low grade dysplasia in patients with Barrett's oesophagus.

Recently, a study combining three forms of tissue spectroscopy demonstrated that the combination of optical techniques can enhance the detection of dysplasia in Barrett's osophagus. ${ }^{13}$ During standard endoscopic procedures, fluorescence spectra were acquired at 11 laser excitation wavelengths between $337 \mathrm{~nm}$ and $620 \mathrm{~nm}$ and one white light (350-750 $\mathrm{nm})$ reflectance spectrum, all in less than one second. Light delivery and collection was mediated via an optical fibre probe passed through the accessory channel of a standard endoscope. The acquired spectra were generated from the uppermost tissue layers, where almost $90 \%$ of all gastrointestinal cancers originate. Fluorescence, reflectance, and LSS were used individually and in combination to evaluate patients for both low grade and high grade dysplasia. Each technique was found to be complementary and contributed critical data to the assessment of precancerous tissue without the use of exogenous dyes or fluorophores. This and other new optical technologies that evaluate tissue in situ are free from the artefacts of biopsy and histological processing and may therefore advance the understanding of malignant transformation.

\section{OPTICAL COHERENCE TOMOGRAPHY}

Optical coherence tomography (OCT) is a method that provides two dimensional cross sectional images of the gastrointestinal tract. Like endoscopic ultrasonography, OCT provides true anatomical images corresponding to the layers of the gastrointestinal tract (mucosa, submucosa, muscularis propria, and serosa/adventitia). However, by using light instead of ultrasound waves, the resolution of OCT is nearly 10-fold greater than that of high frequency endoscopic ultrasonography and approaches that of light microscopy. ${ }^{14-18}$

Measurement of optical back scattering is performed by low coherence interferometry. This method typically uses a low coherence light source such as a superluminescent diode, which has a coherence length of $20 \mu \mathrm{m}$. The light beam is split in two by an optical fibre splitter, with one beam directed to the tissue via an optical fibre and the other beam directed to a mirror located at a precisely controlled distance. The backscattered light from the tissue is combined with the reflected light from the mirror and interference occurs only when the path lengths match to within the $20 \mu \mathrm{m}$ coherence length of the source. By measuring the degree of interference at each mirror position as the mirror is moved, a quantitative measurement of optical backscattering at different depths is obtained. The coherence length of the light source determines the maximal axial resolution that can be obtained. Transverse resolution is determined by the spot size of the focused beam directed at the tissue and the amount that the apparatus is translated at each during the scan; it is typically also about $20 \mu \mathrm{m}$. OCT is typically performed with near infrared light because tissue is relatively transparent at these frequencies. Scattering of light in tissue limits the depth of scanning to about 1 to $2 \mathrm{~mm}$ in the gastrointestinal tract, generally restricting OCT imaging to the mucosa and submucosa when performed during endoscopy.

OCT is typically performed using catheters passed through the accessory channel of standard endoscopes, colonoscopes, or duodenoscopes. Radial scanning and linear scanning catheters have been described; linear systems have generally yielded better images. Unlike endoscopic ultrasound, OCT can be performed through air so tissue contact or coupling is not required. Scanning depth is limited to $1-2 \mathrm{~mm}$ because of scattering of light by tissue. Most of the systems described achieve a resolution of about $20 \mu \mathrm{m}$, which is sufficient for visualising mucosal glands, crypts and villi but not cellular features such as nuclear dysplasia. In contrast, high frequency ultrasound resolution is typically 100-200 $\mu \mathrm{m}$. In newer systems, a 512 by 512 pixel image can be acquired in 0.25 seconds; older systems required several seconds to scan an image, which sometimes lead to blurring from patient motion.

There are abundant potential applications for OCT. ${ }^{19-25}$ In conditions where sampling errors occur, such as endoscopic surveillance of high grade dysplasia in patients with Barrett's oesophagus or ulcerative colitis, OCT could be applied to survey regions of mucosa to locate regions likely to harbour pathology (figs 2 and 3). OCT could conceivably be used to assist in the diagnosis of microscopic inflammatory conditions, such as collagenous or lymphocytic colitis. OCT could also be used to distinguish hyperplastic from adenomatous polyps. Although OCT has advantages over currently available imaging modalities, in its current form, it still has several limitations. The time required to obtain images is long, and image resolution, currently $10-20 \mu \mathrm{m}$, is not sufficient to replace histological diagnosis. Newer, ultra-short pulsed light sources for OCT have the potential to improve axial resolution to $2-4 \mu \mathrm{m} .{ }^{21}$ Just as endoscopic ultrasonography requires the endoscopist to become familiar with radiological techniques, ultrasound imaging artefacts, and three dimensional anatomy, OCT will require the endoscopist to become more knowledgeable regarding histopathology.

The first prospective evaluation of OCT in the oesophagus was recently published. ${ }^{22}$ Three criteria were used to diagnose specialised intestinal metaplasia (Barrett's oesophagus): lack of normal oesophageal or gastric morphology, inhomogeneous tissue contrast, and presence of submucosal glands. The presence of at least two of the three criteria was found to be $97 \%$ sensitive and $92 \%$ specific using histopathology as the standard. However, it is difficult to directly compare these results to previous studies estimating the accuracy of visual identification of intestinal metaplasia on endoscopy because the OCT 

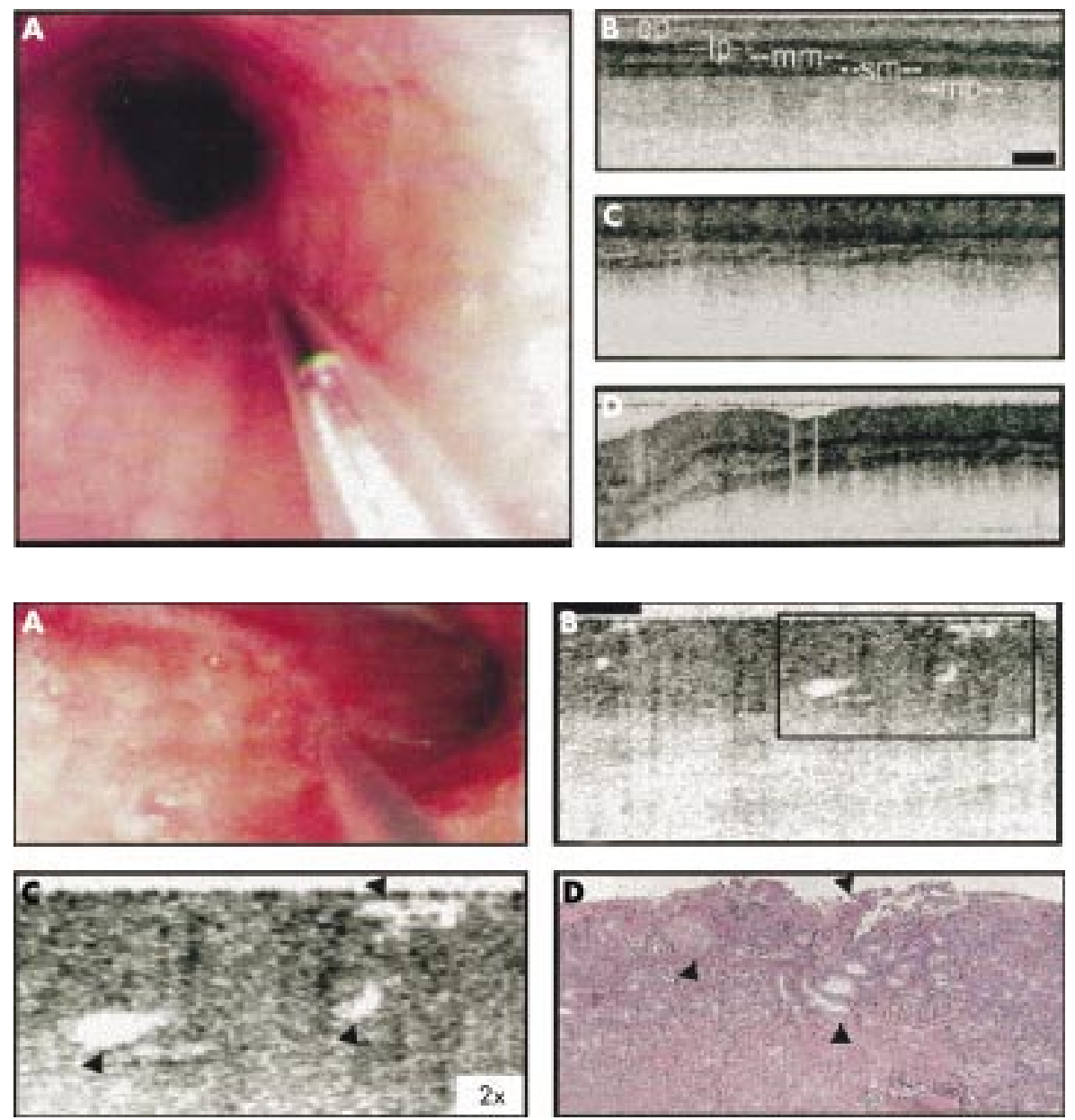

Figure 2 Optical coherence tomography in a patient with a normal oesophagus. (A) endoscopic view of OCT catheter probe in distal oesophagus. (B-D) linear arrangment of mural layers (ep, epitheliaum; lp, lamina propria; mm, muscularis mucosa; sm, submucosa; mp, muscularis propria. From reference 21 : Endoscopy 2000;32:921-30.

Figure 3 Optical coherence tomography in a patient with Barrett's oesophagus. (A) Endoscopic view of OCT catheter probe in distal oesophagus. (B) OCT image demonstrating loss of linearity and demonstration of submucosal glands. (C) Higher magnification of area in box in B. (D) Histological correlation of OCT image. From reference 21 : Endoscopy 2000;32:921-30. study set consisted mainly of normal oesophagus, Barrett's oesophagus, and normal stomach. In practice, the main difficulty in diagnosing intestinal metaplasia visually during endoscopy arises in distinguishing Barrett's from inflammation in the distal oesophagus, and in distinguishing intestinal metaplasia from the less worrisome gastric metaplasia.

In the past five years, "proponents of this technology contended that it could join magnetic resonance imaging (MRI), ultrasound, and computed tomography (CT) as a major imaging modality. At that time, the goals for improving OCT were higher resolution, faster acquisition rates and better system integration software. Also, no large scale clinical trials testing it against other imaging techniques had been performed. Today, components borrowed from the telecommunications arena have improved resolution and image delivery."17 However, commercial systems are still not widely available. Of the many technologies operating under the term, "optical biopsy," OCT remains one of the most promising.

\section{OTHER PROMISING FORMS OF IMAGE ENHANCEMENT}

Raman spectroscopy is another form of image enhancement based on the principle that incident light can cause molecules within a tissue to vibrate and rotate. ${ }^{26}$ The charged molecules can resonate emitting energy that can be measured spectrally. In this form of spectroscopy, light with wavelengths in the ultraviolet or infrared region of the spectrum is used to excite a target tissue. The resulting resonance spectrum represents the tissue's content of specific components such as distinct nucleic acids and proteins. Thus Raman spectroscopy provides the opportunity to obtain a molecular profile or "fingerprint" of a tissue. An in vivo method of Raman spectroscopy has been developed using near infrared wavelengths for excitation. This system uses an optical fibre probe passed through the accessory channel of an endoscope. Reproducible spectra can be obtained even in the presence of blood overlying the tissue being studied. While still in their early stages, clinical studies using Raman spectroscopy to detect gastrointestinal dysplasia are currently in progress. ${ }^{26}$

Confocal microscopy is a well known laboratory technique previously limited to "bench top" use. Recent advances in technology have yielded clinically applicable tools using this technology for both ex vivo tissue analysis and potentially endoscopic applications. ${ }^{27}{ }^{28}$ Inoue et al, studied fresh, untreated mucosal specimens from the oesophagus, stomach, and colon obtained at endoscopy using a laser scanning confocal microscope. ${ }^{28}$ Images were obtained with a scanning time of, on average, less than two seconds and the images compared favourably with those obtained by standard haematoxylin and eosin stained light microscopy (figs 4, 5). Using the nucleus to cytoplasm ratio as a diagnostic criteria for cancer, light scanning confocal microscopy had a diagnostic accuracy of $89.7 \%$ Seminal work by Sabharwal et al using fibre optic bundles has yielded a tool that may be clinically useful. The tool, a confocal microendoscope, has already been used successfully in animal models. ${ }^{27}$

The use of exogenous dyes in conjunction with high magnification endoscopy has long been advocated to enhance endoscopic imaging in both the oesophagus and colon. Recently, newer pharmaceutical agents have been proposed to enhance the ability of even advanced imaging techniques to provide information about tissue chemistry or morphology in vitro and in vivo. ${ }^{29-31}$ Ito et al used a derivative of the dye indocyanine green coupled to antihuman carcinoembryonic antigen as a marker visible via infrared fluorescence imaging to detect 

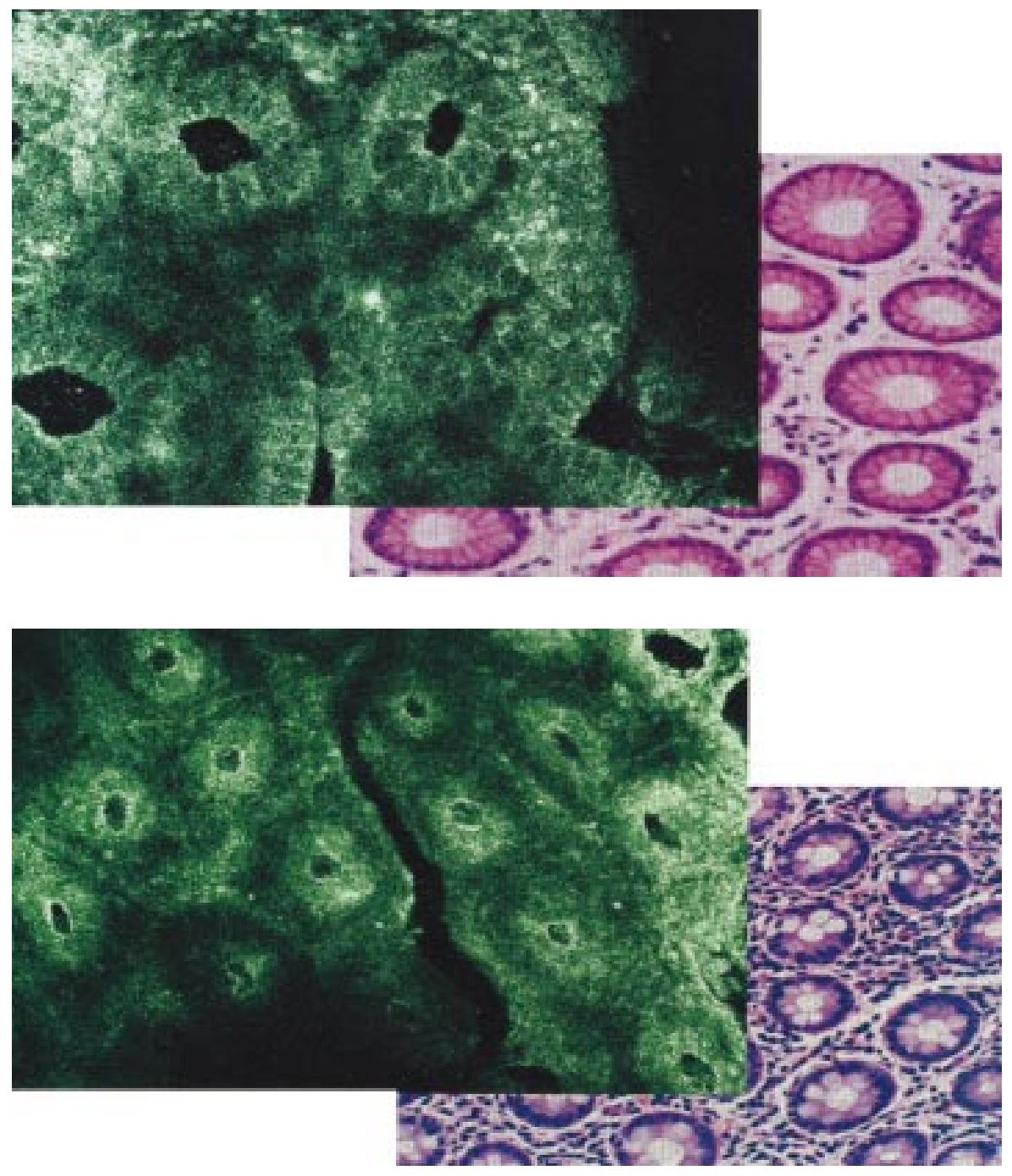

Figure 4 Image of normal gastric mucosal biopsy obtained using confocal microscopy and histological correlate. From reference 28 : Endoscopy 2000;32:439-43.

Figure 5 Image of normal colonic mucosal biopsy obtained using confocal microscopy and histological correlate. From reference 28: Endoscopy 2000;32:439-43. "microlesions" in human gastric mucosa. ${ }^{30}$ In this preliminary study, biopsy specimens from freshly resected stomachs containing gastric cancer were correctly identified via infrared endoscopic imaging. Mayinger et al used 5-aminolevulinic acid (5-ALA) to enhance fluorescence imaging in 22 patients with known or treated malignant and precancerous oesophageal lesions. ${ }^{31}$ Patients were used as their own controls in that biopsy specimens were obtained in each patient under white light and pharmaceutically enhanced conditions. Biopsies directed using only white light endoscopy resulted in a $25 \%$ positive yield. However, the 5-ALA induced fluorescence directed biopsies resulted in an $85 \%$ yield. The authors concluded that 5-ALA induced fluorescence endoscopy may be superior to white light endoscopy for the detection of cancerous or dysplastic oesophageal lesions.

\section{SUMMARY}

The limitations of standard endoscopic practice are rapidly being overcome by advanced methods. Technological achievements in imaging are currently being tested in clinical practice and may soon yield tools capable of making real time tissue diagnoses at endoscopy or directing endoscopic biopsies to previously unrecognised tissue abnormality. Although not yet available for routine use by most endoscopists, the rate of technological advancement suggests that at least one or more forms of enhanced endoscopic imaging should be available within the foreseeable future.

\section{REFERENCES}

1 Kapadia C, Cutruzzola F, O’Brien F, et al. Laser-induced fluorescence spectroscopy of human colonic mucosa. Gastroenterology 1990;99:150-7.

2 Cothren RM, Richards-Kortum R, Sivak MV Jr, et al. Gastrointestinal tissue diagnosis by laser-induced fluorescence spectroscopy at endoscopy. Gastrointest Endosc 1990;36:105-11

3 Cothren R, Sivak M, Van Dam J, et al. Detection of dysplasia at colonoscopy using laser-induced fluorescence: a blinded study. Gastrointest Endosc 1996:44: 168-76.

4 Panjehpour M, Overholt BF, Schmidhammer JL, et al. Spectroscopic diagnosis of esophageal cancer: new classification model, improved measurement system. Gastrointest Endosc 1995;41:577-81.

5 Panjehpour M, Overholt BF, Vo-Dinh T, et al. Endoscopic fluorescence detection of high-grade dysplasia in Barrett's esophagus. Gastroenterology 1996;111:93-101.

6 Romer T, Fitzmaurice M, Cothren R, et al. Laser-induced fluorescence microscopy of normal colon and dysplasia in colonic adenomas: implications for spectroscopic diagnosis. Am J Gastroenterol 1995;90:81-7

7 Wang TD, Van Dam J, Crawford JM, et al. Fluorescence endoscopic imaging of human colonic adenomas. Gastroenterology 1996;111:1182-91.

8 Wang TD, Crawford JM, Feld MS, et al. In vivo identification of colonic dysplasia using fluorescence endoscopic imaging. Gastrointest Endosc 1999;49:447-55

9 Perelman LT, Backman V, Wallace M, et al. Observation of periodic fine structure in reflectance from biologic tissue: a new technique for measuring nuclear size distribution. Phys Rev Lett 1998;80:627-30.

10 Zonios G, Perelman LT, Backman V, et al. Diffuse reflectance spectroscopy of of human adenomatous colon polyps in vivo. Applied Optics 1999;38:6628-37

11 Backman V, Perelman LT, Wallace MB, et al. Clinical diagnosis of precancerous and cancerous changes in human epithelial tissues with light-scattering spectroscopy. Nature 2000;406:35-6.

12 Wallace MB, Perelman LT, Backman V, et al. Endoscopic detection of dysplasia in patients with Barrett's esophagus using light-scattering spectroscopy. Gastroenterology 2000;1 19:677-82. 
13 Georgakoudi I, Jacobson BC, Van Dam J, et al. Fluorescence, reflectance, and light scattering spectroscopy for evaluating dysplasia in patients with Barrett's esophagus. Gastroenterology 2001;120:1620-9.

14 Tearney GJ, Brezinski ME, Bourna BE, et al. In vivo endoscopic optical biopsy with optical coherence tomagraphy. Am J Gastroenterol 1997:92:1800-4.

15 Tearney GJ, Brezinski ME, Southern JF, et al. Optical biopsy in human gastrointestinal tissue using coherence tomagraphy. Am J Gastroenterol 1997:92:1800-4.

16 Kobayashi K, Izatt JA, Kulkarni MD, et al. High resolution cross-sectional imaging of the gastrointestinal tract using optical coherence tomography: preliminary results. Gastrointest Endosc 1998;47:515-23.

17 Reiss SM. OCT update: pushing a promising technology to market. Biophotonics International 2001;40-5.

18 Fujimoto J, Brezinski $M$, Tearney $G$, et al. Optical biopsy and imaging using optical coherence tomography. Nat Med 1995;1:970-2.

19 Bouma B, Tearney G, Compton C, et al. High-resolution imaging of the human esophagus and stomach in vivo using optical coherence tomography. Gastrointest Endosc 2000;51:467-74.

20 Jaeckle S, Gladkova N, Terentieva A, et al. In vivo endoscopic optical coherence tomography of the human gastrointestinal tract- toward optical biopsy. Endoscopy 2000;32:743-9.

21 Li X, Boppart SA, Van Dam J, et al. Optical coherence tomography: advanced techniques for endoscopic imaging of Barrett's esophagus. Endoscopy 2000;32:921-30.

22 Poneros J, Brand S, Bouma B, et al. Diagnosis of specialized intestinal metaplasia by optical coherence tomography. Gastroenterology 2001;120:7-12

23 Seitz U, Jaeckle S, Feldchtein F, et al. First in vivo optical coherence tomography in the human bile duct. Endoscopy 2001;33:1018-21.
24 Sivak M, Kobayashi K, Izatt J, et al. High-resolution endoscopic imaging of the $\mathrm{Gl}$ tract using optical coherence tomography. Gastrointest Endosc 2000;51:474-9.

25 Zuccaro G, Gladkova N, Vargo J, et al. Optical coherence tomography of the esophagus and proximal stomach in health and disease. Am J Gastroenterol 2001;96:2633-9.

26 Shim M, Song L, Marcon N, et al. In vivo near-infrared Raman spectroscopy: demonstration of feasibility during clinical gastrointestinal endoscopy. Photochem Photobiol 2000;72: 146-50.

27 Sabharwal YS, Rouse AR, Donaldson L, et al. Slit-scanning confocal microendoscope for high-resolution in vivo imaging. Applied Optics 1999;38:7133-44.

28 Inoue $\mathbf{H}$, lgari T, Nishikage T, et al. A novel method of virtua histopathology using laser-scanning confocal microscopy in vitro with untreated fresh specimens from the gastrointestinal mucosa. Endoscopy 2000;32:439-43

29 Messmann H, Kullmann F, Wild T, et al. Detection of dysplastic lesions by fluorescence in a model of colitis in rats after previous photosensitization with 5-aminolaevulinic acid. Endoscopy 1998;30:333-8.

30 Ito S, Muguruma N, Kusaka $Y$, et al. Detection of human gastric cancer in resected specimens using a novel infrared fluorescent anti-human carcinoembryonic antigen antibody with an infrared fluorescence endoscope in vitro. Endoscopy 2001;33:849-53.

31 Mayinger B, Neidhardt S, Reh H, et al. Fluorescence induced with 5-aminolevulinic acid for the endoscopic detection and follow-up of esophageal lesions. Gastrointest Endosc 2001;54:572-8. 